\title{
Collaboration between academics and industry in clinical trials: cross sectional study of publications and survey of lead academic authors
}

\author{
Kristine Rasmussen, ${ }^{1}$ Lisa Bero, ${ }^{2}$ Rita Redberg, ${ }^{3}$ Peter C Gøtzsche, ${ }^{1}$ Andreas Lundh ${ }^{4}$
}

${ }^{1}$ Nordic Cochrane Centre,

Rigshospitalet, Copenhagen, Denmark

${ }^{2}$ Charles Perkins Centre and Faculty of Pharmacy, University of Sydney, Sydney, NSW, Australia

${ }^{3}$ UCSF Division of Cardiology, San Francisco, CA, USA

${ }^{4}$ Centre for Evidence-Based Medicine Odense (CEBMO) and Odense Patient data

Exploratory Network (OPEN),

Odense University Hospital,

Odense, Denmark

${ }^{5}$ Department of Clinical

Research, University of

Southern Denmark, Odense,

Denmark

${ }^{6}$ Department of Infectious

Diseases, Hvidovre Hospital, Hvidovre, Denmark

Correspondence to: K Rasmussen Kristine.rasmussen12@imperial. ac.uk

Additional material is published online only. To view please visit the journal online.

Cite this as: $B M J$ 2018;363:k3654 http://dx.doi.org/10.1136/bmj.k3654

Accepted: 30 July 2018

\section{ABSTRACT}

OBJECTIVES

To determine the role of academic authors, funders, and contract research organisations in industry funded trials of vaccines, drugs, and devices and to determine lead academic authors' experiences with industry funder collaborations.

DESIGN

Cross sectional analysis of trial publications and survey of lead academic authors.

\section{ELIGIBILITY CRITERIA FOR SELECTING STUDIES}

The most recent 200 phase III and IV trials of vaccines, drugs, and devices with full industry funding, at least one academic author, published in one of the top seven high impact general medical journals (New England Journal of Medicine, Lancet, JAMA, BMJ, Annals of Internal Medicine, JAMA Internal Medicine, and PLoS Medicine).

\section{RESULTS}

Employees of industry funders co-authored 173 (87\%) of publications; 183 (92\%) trials reported involvement of funders in design, and 167 (84\%) reported involvement of academic authors. Data analysis involved the funder in $146(73 \%)$ trials and the academic authors in 79 (40\%). Trial reporting involved the funder in $173(87 \%)$ trials and academic authors in 197 (99\%). Contract research organisations were involved in the reporting of 123 $(62 \%)$ trials.

Eighty (40\%) of 200 lead academic authors responded to the survey. Twenty nine (33\%) of the 80 responders reported that academics had final say on the design. Ten responders described involvement of an unnamed funder and/or contract research organisation employee in the data analysis and/or reporting. Most academic authors found the collaboration with industry funder beneficial, but $3(4 \%)$ experienced delay in publication due to the industry funder and 9 (11\%) reported

\section{WHAT IS ALREADY KNOWN ON THIS TOPIC}

The biomedical industry funds most clinical trials

Industry funders may influence how trials are designed and reported, sometimes serving financial rather than public interest

\section{WHAT THIS STUDY ADDS}

In most industry funded trials reported in high impact medical journals, all aspects of the clinical trial were influenced by the industry funder

Reported access to data does not always mean access to the entire trial dataset Lead academic authors involved in industry funded trials found the collaboration with the funder beneficial, but some reported loss of academic freedom

disagreements with the industry funder, mostly concerning trial design and reporting.

\section{CONCLUSIONS}

Industry employees and academic authors are involved in the design, conduct, and reporting of most industry funded trials in high impact journals. However, data analysis is often conducted without academic involvement. Academics view the collaboration as beneficial, but some report loss of academic freedom.

\section{Introduction}

Collaboration between industry and academics is common in the development of vaccines, drugs, and devices, as it can be mutually beneficial. The academics provide access to trial participants and clinical and methodological expertise, and industry provides funding and expertise. The degree of independence and the roles of academics and industry vary across trials. Trials may be run by academic trial units independently but with unrestricted industry funding, or the only contribution from industry could be free provision of study drugs.10 Alternatively, academics may be involved in trials as honorary authors to give a trial scientific credibility and downplay the role of the industry funder. ${ }^{34}$

Previous work and examples suggest that collaboration between academics and industry may result in commercial considerations outweighing science and constraints on academic freedom. For example, previous studies have found that some trial agreements permit funders to block publication. ${ }^{5-8}$ Furthermore, academics have reported problems with stalling of publications, restriction of publication rights, and threats to never fund their institution again owing to reporting of negative results or harms. ${ }^{9} \mathrm{~A}$ survey of Canadian trial investigators found problems in relation to industry control over study design, ownership of and access to data, and data analysis. ${ }^{10}$

Although most clinical drug and device trials are funded by industry, the nature of the collaboration between industry and academics has received little attention. ${ }^{11}$ Previous studies have considered the question on a general level. ${ }^{5-10} 12$ To our knowledge, no studies have surveyed the role and practices of academics, industry, and contract research organisations (CROs) when collaborating in relation to specific clinical trials of vaccines, drugs, or devices. Our objectives were to determine the role of academic authors, funders, and CROs in industry funded trials of vaccines, drugs, and devices and to determine lead academic authors' experiences with industry funder collaborations. 


\section{Methods}

This study was in two parts and was based on a protocol written before the conduct of the study. The first part of the study was a cross sectional study of trial publications, and the second part was a survey of the lead academic authors of the 200 most recent industry funded trials of vaccines, drugs, and devices published in the top seven high impact general medical journals. We started with descriptive and quantitative data collection from trial publications and built on that with a survey of lead academic authors. We used the responses from the survey to further explore and explain the results from the trial publications.

\section{Cross sectional study}

Search

One author (KR) manually searched the top seven general and internal medical journals according to the impact factor of the 2015 Journal Citation Report (the New England Journal of Medicine (NEJM), the Lancet, $J A M A$, the BMJ, the Annals of Internal Medicine, JAMA Internal Medicine, and PLoS Medicine) for the most recent trial publications meeting our inclusion criteria. ${ }^{13}$ The search started from April 2017 and went backwards in time until 200 studies were included.

\section{Inclusion criteria}

We included publications of phase III and IV trials with one or more academic authors (determined using the institutional address) that disclosed full industry funding. We defined full industry funding as any trial with funding exclusively from one or more vaccine, pharmaceutical, or device companies. We included industry supported trials described as investigator initiated if the only funding was from industry sources. ${ }^{14}$

We defined academic authors as authors whose affiliation in the publication was a clinic, hospital, university, or non-profit academic research centre. We selected the lead academic author according to the following rank: corresponding author, first author, last author, second author, third author, etc. If a publication had more than one academic corresponding author, we deemed the lead academic author according to the following rank: first author, last author, second author, third author, etc.

\section{Exclusion criteria}

We excluded trials of fluid therapy and dietary supplements, trials with mixed funding (public and industry funded, including free provision of study drug or device only), and secondary publications (for example, subgroup analysis). If two publications of the same trials were identified (for example, an interim analysis and the planned analysis), we included only the most recent publication. If a lead academic author had published more than one eligible trial, we included only the most recent trial. A second author (AL) confirmed that the 200 trial publications met the inclusion and exclusion criteria. Disagreements were resolved by discussion.

\section{Data extraction}

For each trial publication, two authors (AL, KR) independently extracted data into a standardised data sheet. We extracted data on the role of the academic authors, funder, and CRO with regard to trial design, conduct, data analysis, and reporting. Academic authors were all employed by clinics, hospitals, and/ or universities. We defined a funder employee as an author or acknowledged person who had the funding company listed as affiliation and a CRO employee as an author or acknowledged person who had a CRO listed as affiliation. We defined a CRO as a commercial company that provides research services to the funder in relation to certain parts of the trial design, conduct, analysis, or reporting. This definition included people who were hired by the funder as statistical consultants or medical writers.

We also extracted data from the publications on access to data, trial agreements, and the lead academic author's conflicts of interest as disclosed in the included paper. If the publication did not report conflicts of interest, we retrieved information from the International Committee of Medical Journal Editors (ICMJE) disclosure form. We focused on conflicts of interest outside the submitted work, as most lead academic authors were investigators and we wanted to identify the group of academic authors with more extensive funder collaboration. Therefore, we did not count being an investigator or receiving a grant from the trial funder in relation to the included trial as a conflict of interest (see appendix 1 for full details of the fields extracted). In this study, we used the ICMJE definition of a conflict of interest: "A conflict of interest exists when professional judgment concerning a primary interest (such as patients' welfare or the validity of research) may be influenced by a secondary interest (such as financial gain)."15 Disagreements were resolved by discussion.

When we started the data extraction, we found that the contribution of individual authors and role of the funder were often not clear from the publications in the NEJM, as roles were typically described in broad terms (for example, the trial was designed by a steering committee of academics and funder representatives, but the publication did not describe which authors were members). We therefore decided to include supplementary material (such as lists of steering committee members and contributions statements) for data extraction of $N E J M$ publications, if available.

\section{Survey}

We designed our survey by using Qualtrics (www. qualtrics.com). Each lead academic author was tracked by a unique identifier to allow matching of the survey response to the publication and to track authors who had not responded. The identity of survey authors was available to only two of the authors of our study (AL and $\mathrm{KR}$ ), and we did not link any quotes to individual identifiers, so the rest of the authors and the public could not match responses with specific authors. 


\begin{tabular}{|c|c|}
\hline & No (\%) \\
\hline \multicolumn{2}{|l|}{ Journal } \\
\hline New England Journal of Medicine & $106(53)$ \\
\hline Lancet & $62(31)$ \\
\hline JAMA & $25(13)$ \\
\hline Annals of Internal Medicine & $4(2)$ \\
\hline JAMA Internal Medicine & $2(1)$ \\
\hline BMJ & $1(1)$ \\
\hline \multicolumn{2}{|l|}{ Intervention } \\
\hline Drug & $165(83)$ \\
\hline Device & $26(13)$ \\
\hline Vaccine & $9(5)$ \\
\hline \multicolumn{2}{|l|}{ Comparator } \\
\hline Active treatment & $79(40)$ \\
\hline Multiple arms (active treatment and placebo) & $27(14)$ \\
\hline Placebo or no additional treatment & $94(47)$ \\
\hline \multicolumn{2}{|l|}{ Authorship } \\
\hline Academic and industry funder authors* & $173(87)$ \\
\hline Academic and CRO authors & $5(3)$ \\
\hline Solely academic authors & $22(11)$ \\
\hline First author academic & $196(98)$ \\
\hline First author funder & $4(2)$ \\
\hline Last author academic & $154(77)$ \\
\hline Last author funder & $42(21)$ \\
\hline Last author CRO & $2(1)$ \\
\hline Last author othert & $2(1)$ \\
\hline Corresponding author academic & $192(96)$ \\
\hline Corresponding author funder & $8(4)$ \\
\hline \multicolumn{2}{|c|}{ Lead academic author's reported conflicts of interest } \\
\hline Conflict(s) of interest with funder $\ddagger$ & $165(83)$ \\
\hline Conflict(s) of interest with other company & $14(7)$ \\
\hline No conflicts of interest & $21(11)$ \\
\hline \multicolumn{2}{|l|}{$C R O=$ contract research organisation. } \\
\hline \multicolumn{2}{|c|}{$\begin{array}{l}\text { * Some trials also had CRO employed co-authors. } \\
\text { t Refers to author employed by industry company other than industry } \\
\text { funder and one author with unclear affiliation (CRO or private clinic). } \\
\text { ‡ Those who had conflicts of interest with funder could also have } \\
\text { conflicts of interest with other industry companies. Grant from funder or } \\
\text { being investigator in included trial did not count as conflict of interest. }\end{array}$} \\
\hline
\end{tabular}

classified as independent trials (that is, all aspects of the industry funded trial were carried out by academic authors without involvement of the funder or a CRO). In four of these eight trials, the lead academic author had disclosed conflicts of interest with the funder that were outside the submitted work.

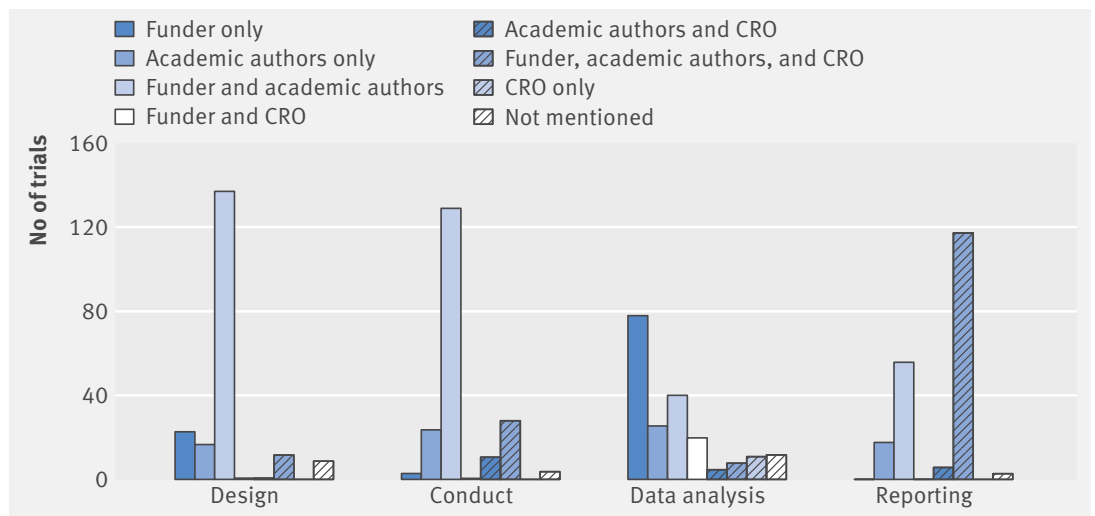

Fig 2 | Reported involvement in 200 trials. $C R O=$ contract research organisation
The data analysis was done by a median of 3 (range 1-31) authors with the involvement of a median of 1 (0-11) funder employee. In 95 trial publications, we were unable to identify the exact number of authors involved in data analysis. Although we aimed to extract data on who did the actual statistical analysis, this was often difficult as publications used phrases such as "All authors analysed and interpreted the data," even though a publication might have 18 authors. Other publications used a phrase from the ICMJE requirements for authorship, describing individual authors' involvement in "acquisition, analysis, or interpretation of data," and thus grouped three very different processes making it impossible to assess who did the statistical analysis. ${ }^{15}$ In 10 trials, the funder was stated as having no role in the data analysis, but who else was involved was unclear (for example, a CRO).

\section{Data access and trial agreements}

All authors declared access to data in 77 (39\%) trials, and some academic authors did so in an additional 67 (34\%) trials. For the remaining trials, 52 (26\%) stated that authors vouched for the data without specifying who had access and 4 (2\%) had no description of data access. Only $9(6 \%)$ of 144 trials reporting data access specified the type of data: 2 (1\%) reported access to raw data, $3(2 \%)$ access to analysed data only, 1 (1\%) access to summary data for all patients, but only individual patient data from own institution, and 3 $(2 \%)$ access to data used in the publication.

In $16(8 \%)$ trial publications, confidentiality agreements with the funder were described, and $4(2 \%)$ publications described that no such agreements existed. For the remaining 180 (90\%) trials, the publication did not mention confidentiality agreements.

\section{Survey}

We received responses from 106 (53\%) lead academic authors (fig 1). Three lead academic authors were unreachable by email, post, and telephone. Eighty (40\%) of 200 responded to survey questions, 10 (5\%) accessed the survey without providing any response, and $16(8 \%)$ replied that they did not want to participate (appendix 3). Our comparison of trials' and authors' characteristics between those who responded to those who did not showed no important differences (appendix 4).

\section{Decisions on and involvement in trial design,} analysis, and reporting, as reported by lead academic authors

Academic authors were involved in the choice of comparator treatment in most trials, whereas funders were involved in choice of outcomes in most trials (appendix 5). Twenty nine (33\%) of responders reported that academics had final say on the design. See figure 3 for all responses, box 1 for selected authors' comments, and appendix 6 for additional authors' comments. 


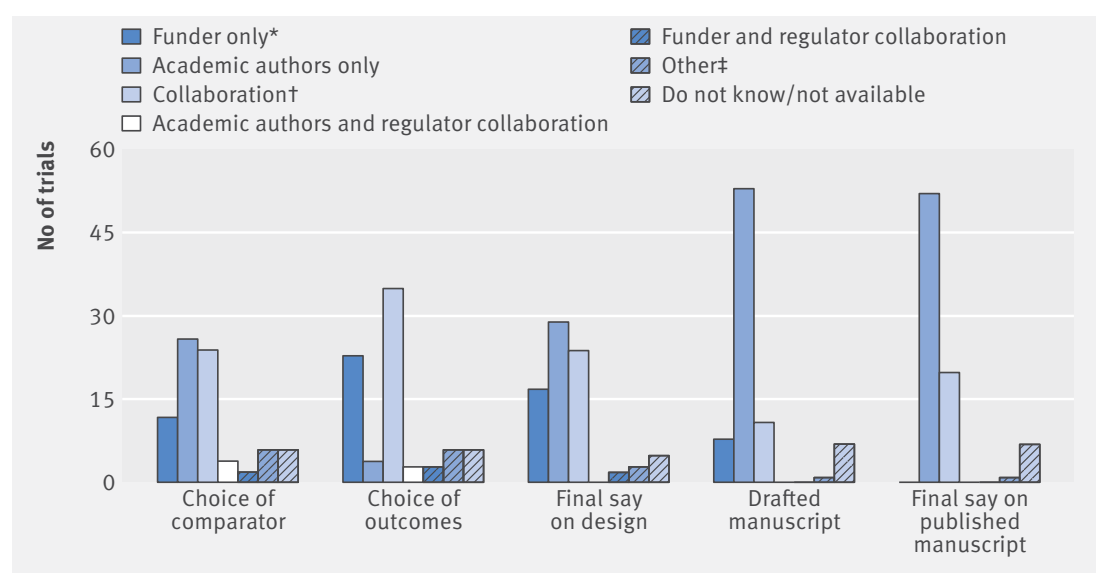

Fig 3 | Reported involvement according to survey. $\mathrm{CRO}=$ contract research organisation. *Funder and CRO also includes unacknowledged people for conducted statistical analysis and drafting of manuscript. +Collaboration includes academic, funder, and/or CRO; academic, funder, and/or regulator; academic and CRO; funder and CRO. ¥Other includes regulator, journal, unknown, or unclear. No responders reported CRO as the only actor for any category
Ten $(13 \%)$ responders described involvement of an unnamed funder, CRO employee, or both in the data analysis and/or reporting. An additional 7 (9\%) responders described funder and/or CRO employee involvement in trial design, data analysis, or reporting that was not reported in the corresponding publication.

\section{Trial agreements and data access}

Sixty three (79\%) responders reported signing a trial agreement with the funder (table 2). Eight of these commented that the contract theysigned gave the funder the right to review and comment on the manuscript and presentations but did not give the funder approval rights. However, six of these responders' eight trials had funder employed co-authors, thereby indirectly giving funder approval right (appendices 6 and 7), and one of these authors also described disagreements with the funder about the content of the manuscript. Only $4(6 \%)$ of the 63 trials with agreements had a description of a confidentiality agreement in the trial publication. Another trial publication reported that no confidentiality agreement existed, but the survey contradicted this and described that the data was $100 \%$ embargoed before publication suggesting the existence of a confidentiality agreement.

Most of the lead academic authors reported access to the entire dataset (table 2). Seventy three of the trials allowed for a comparison of access to data between survey responses and statements in trial publications. In $5(7 \%)$ trials, the lead academic author had access to data according to the publication, but they denied such access in the survey. In three trial publications that used the phrase "The authors vouch for the data," the lead academic author did not have access to the data according to the survey.

\section{Experience with collaboration}

Only a few responders reported problems with the funder, and the most common problems were delays in publication and disagreements with the funder, mostly concerning trial design and reporting (table 2). Disagreements were generally described as minor (see box 1 and appendix 6 for detailed comments).

The most commonly reported benefit of collaborating with the industry funder was funding, reported by $33(41 \%)$ responders. Thirteen (16\%) responders found the fact that they were contributing new research a benefit of the collaboration. Eight (10\%) responders reported the trial publication and subsequent publications using the same dataset as a benefit of the collaboration (box 1 and appendix 6). In our subgroup analyses, we did not find any differences in characteristics of trials or responses to the survey in relation to conflicts of interest, type of intervention, industry involvement, and collaboration status (appendix 7).

reported CRO involvement in reporting, whereas the corresponding publication did not describe this involvement. Fifty two (65\%) responders reported that academic authors solely had final say on the published manuscript. However, 40 of these 52 trials included funder employed co-authors.

\section{Discussion}

We found that the funders of industry funded trials were usually involved in every step of the trial. Nevertheless, 


\section{Box 1: Selected comments in response to survey questions}

Selected comments for benefits of collaborating with the trial industry funder

Publication- "The Journal X paper! And more to come"; "Opportunity to author papers of important study results in high impact journals"

Personal benefits- "Being PI of a positive trial is always a benefit in CV and recognition"; "...That being said, I would not object to all industry-sponsored studies being listed as 'the Trial XInvestigators' or something similar-ultimately, despite our input, these are studies done by the company"

Selected comments for confidentiality agreement

Confidential until publication - "No for results which are already in the public domain, obviously, but yes for all other unpublished data"

Miscellaneous - "The agreement stated that sponsor could request up to 30 days confidentiality if necessary for patent protection"

Selected comments for choice of comparator

Collaboration- "The company has to obviously agree with the study design. They fund the trial"

Miscellaneous- "The sponsor did not want a placebo arm in the trial. We insisted upon it for safety evaluation and they ultimately agreed to a placebo arm with deferred treatment"

Selected comments for final say in study design

Funder - 'We advised the sponsor study team (extensively) and I would generally say that they listened, but they did not 'require' sign-off from the steering committee before finalizing the protocol so in that sense, they had final say"

\section{Selected comments for statistical analysis}

Miscellaneous - "We analyse with independent academic and with the funder statisticians. Sometimes we have to rely on only the funder statisticians which is less satisfactory. However SAPs and protocols are submitted to regulatory and editors before analysis and publication"

\section{Selected comments for access to data}

Miscellaneous - "Not the ENTIRE dataset, but much of it and we were encouraged to ask for specific analyses"; "I had access to all the data but did not have access to the database. E.g., all analyses conducted in sponsor's dataset. Any analysis that we wanted was done"

Selected comments for using data access

Miscellaneous - "I anticipate some 30 high-quality papers will be published using this data set. 12 already published, 4 submitted and 10 currently in preparation"

\section{Selected comments for drafting the manuscript}

Academics writing intro and discussion - "I drafted the introduction and discussion sections, while the company scientific writers drafted the methodology and results sections"

\section{Selected comments for how disagreements with funder were handled by the funder}

Miscellaneous-"Want to postpone and change the wording"

Selected comments from additional comments

Miscellaneous - "Further report not approvable or not approved by the sponsor"

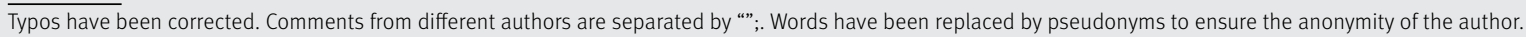

the role of academic authors, funders, and CROs varied greatly. Generally, both academic authors and funders were involved in the trial design, conduct, and reporting. However, the actual data analysis was most often done by funder or CRO employees. Our study showed inaccuracy of published descriptions of authors' roles and access to data. Few industry funded trials were completely independently conducted by academics, and sometimes industry involvement was downplayed or omitted in trial publications. The lead academic authors often found the collaboration beneficial, particularly in relation to funding of the trial. However, some academic authors reported disagreements with the funder, mostly concerning trial design and reporting.

\section{Context of findings}

Lundh et al analysed trials published in the Lancet in 2008-9 and had access to trial protocols. ${ }^{12}$ Our findings of extensive involvement of industry funders and that the roles of funders and CROs were sometimes downplayed in the trial publications are similar to the findings of Lundh et al. Data are often stored and owned by the industry funder; similarly to Lundh et al, we found that trial publications rarely described what type of data the academic authors had access to and whether they used this access. ${ }^{18} 19$ We believe that descriptions of data access in publications may not be accurate. In 67 of the 69 Lancet protocols, no information was given on academic authors' access to data, in striking contrast to the papers, which indicated that one or more academic authors had access to the data in 64 trials. $^{12}$

Ghost authorship, whereby people are involved in important aspects of clinical trials without the involvement being disclosed in the publications, has been well described. ${ }^{4} 102021$ We found evidence of ghost authorship in 17 of 80 trials, but this is likely to be an underestimate as some lead academic authors had a small role in the conduct, analysis, and reporting of the trial. Thus, "ghost authoring" could have taken place without the lead academic authors being aware. Gøtzsche et al found a much higher prevalence of $75 \%$ of ghost authorship in trials, particular in 


\begin{tabular}{|c|c|c|c|}
\hline & Yes & No & $\begin{array}{l}\text { Do not know or } \\
\text { not available }\end{array}$ \\
\hline \multicolumn{4}{|l|}{ Trial agreement } \\
\hline Signed trial agreement with industry funder & $63(79)$ & $16(20)$ & $1(1)$ \\
\hline Signed trial agreement included publication agreement† & $46(73)$ & $12(19)$ & $5(8)$ \\
\hline Signed trial agreement included presentation agreement† & $33(52)$ & $21(33)$ & $9(14)$ \\
\hline Signed trial agreement included confidentiality agreement† & $39(62)$ & $19(30)$ & $5(8)$ \\
\hline \multicolumn{4}{|l|}{ Data access } \\
\hline Had access to entire trial dataset & $63(79)$ & $9(11)$ & $8(10)$ \\
\hline Access used by those with access to entire datał & $56(89)$ & $6(10)$ & $1(2)$ \\
\hline \multicolumn{4}{|l|}{ Problems } \\
\hline Delay in publication due to funder & $3(4)$ & $70(88)$ & $7(9)$ \\
\hline Disagreements with funder & $9(11)$ & $64(80)$ & $7(9)$ \\
\hline \multicolumn{4}{|l|}{ Collaboration } \\
\hline Already collaborating with funder/would in future & $67(84)$ & $1(1)$ & $12(15)$ \\
\hline
\end{tabular}

relation to data analysis. ${ }^{20}$ However, prevalence of ghost authorship may have diminished over time, as we found that $87 \%$ of trials had industry co-authors compared with $64 \%$ in the study by Gøtzsche et al. ${ }^{20}$ The ICMJE recommends that those who do not fulfil the authorship criteria but have contributed to the study or publication are acknowledged in the publication. ${ }^{15}$ Therefore, the 17 trials identified from the survey in which people contributed to the statistical analysis or writing of the manuscript were in direct breach of the ICMJE recommendations by not listing them as authors or contributors in the trial publication.

Some academic authors reported that having a high impact publication was a benefit of the collaboration, and academic authors were often (96\%) prominently placed as corresponding authors. Apart from the financial gain from industry collaboration, our results suggest that the collaboration may also lead to nonfinancial or indirect financial gain for academic authors-for example, through authorship of a paper in a high impact journal and authorship of subsequent secondary publications thereby influencing tenure, salary, and grant applications. ${ }^{22}$ Future studies should quantify the magnitude of this type of conflict of interest.

\section{Strengths and weaknesses of study}

To our knowledge, this is the first study to directly survey a large cohort of international academic authors involved in industry funded trials published in high impact general medical journals. Furthermore, no studies have obtained and analysed the input of academic authors regarding their experience with industry funded trials, so this study provides novel insights. Our use of data from both trial publications and survey responses allowed us more comprehensive data compared with studies solely based on a single information source. ${ }^{810}$ A major strength of our study is the fact that we included trials from all over the world, so our results are applicable at an international level. Furthermore, the format of the survey, whereby individual authors could not be identified by the public, is likely to have provided us with more truthful responses, especially with regard to sensitive topics.

Our findings may be somewhat limited by the retrospective nature of the survey. However, we included only the most recent trials, so the authors are more likely to remember the details compared with studies focusing on the past experience of academics in general. ${ }^{10}$ Another possible limitation is the mixture of industry funded trials initiated by academics and company trials initiated for regulatory purposes. From trial publications, we could not completely distinguish between these types of trials, and funder involvement is likely more pronounced in industry funded trials conducted for regulatory purposes. We included trials from high impact journals, of which most were published in the NEJM and Lancet. Thus, the generalisability to smaller journals and specialty journals could be limited. Furthermore, our prevalence of conflicts of interest is likely to be a conservative estimate as it was based on disclosures in journals. Previous studies have found that such conflicts are often under-reported in publications, so the actual figures may be even higher. ${ }^{22-24}$ Additionally, we assessed the supplementary material only for trials published in the NEJM. Academic authors with industry ties are more likely to be favourable towards industry involvement in research than those without ties, so our survey results may show this bias. ${ }^{25}$ Although our survey of academic authors gave valuable insights into the benefits and problems related to industry-academic collaboration, future research could consider taking a more qualitative approach to gain additional understanding of the motives of academic authors. Finally, despite many reminders we received survey responses from only 80 (40\%) academic authors, which was somewhat lower than previous surveys of academic authors. ${ }^{10} 18$ However, we dealt with a sensitive topic and surveyed academics who are quite busy. Previous studies on sensitive topics have found response rates lower than or comparable to ours. ${ }^{26-28}$ The non-responders who declined via email primarily declined owing to lack of time or lack of interest in participating. Furthermore, the characteristics of trials and authors did not differ between responders and non-responders, suggesting that the included responses were representative of the overall group of included academic authors.

\section{Meaning of findings}

We found room for more accurate reporting of authors' contribution in industry funded trials published in high impact general medical journals. A solution could be that guidelines such as ICMJE and CONSORT specifically require authors and funders to report their involvement in key trial components, particularly related to analysis and ownership of and access to data. ${ }^{15} 29$ To improve the accuracy of disclosures of funders' contributions, the disclosure should name everyone conducting the statistical analysis and their affiliation, aswell as discriminatebetween thosewhodid the actual analysis and those who merely participated 
in data acquisition and interpretation. In addition, publications should provide precise information about who holds and owns the entire dataset as well as who had access to it. Furthermore, ethics committees should review complete descriptions of the roles of funders and academic authors and consider them in their deliberations. More accurate reporting of contributorship should give patients greater confidence in trial results and conclusions. Furthermore, journals could refuse publication when these elements are not clearly reported. The presence of ghost authorship could perhaps be reduced by asking the authors to confirm and sign a statement that all contributors to their study have been acknowledged and correcting papers with evidence of ghost authorship. Journals could also penalise all authors of ghost written papers by banning them from future publication. Trials from high impact journals have important effect on clinical decisions. ${ }^{3031}$ Nevertheless, only a few of our included trials had independent analysis. However, academics can demand control over design, data storage, and full data ownership, analysis, and reporting, thereby improving independence and greater reliability of trial results.

\section{Conclusions}

Industry employees and academic authors are involved in the design, conduct, and reporting of most industry funded trials in high impact journals. However, the data analysis was often conducted without academic involvement. Academics view the collaboration with the industry funder as beneficial, but some report loss of academic freedom.

Contributors: KR had the idea for the study and developed the protocol with contributions from all authors. KR and AL extracted data and analysed the results. KR drafted the manuscript. All authors interpreted results, were involved in the reporting of the study, and approved the final version of the manuscript. The corresponding author attests that all listed authors meet authorship criteria and that no others meeting the criteria have been omitted. KR is the guarantor; she accepts full responsibility for the work and the conduct of the study, had access to the data, and controlled the decision to publish.

Funding: No external funding received. Study was financed by institutional salaries.

Competing interests: All authors have completed the ICMJE uniform disclosure form at www.icmje.org/coi_disclosure.pdf (available on request from the corresponding author) and declare: no support from any organisation for the submitted work: RFR is editor of JAMA Internal Medicine, which is included in the sample of journals, but had no role in data extraction or analysis of the results; no financial relationships with any organisations that might have an interest in the submitted work in the previous three years; no other relationships or activities that could appear to have influenced the submitted work.

Ethical approval: The Regional Committee on Health Research Ethics and the Danish Data Protection Agency both confirmed that this survey does not require any form of approval according to Danish law. Transparency statement: The manuscript's guarantor affirms that the manuscript is an honest, accurate, and transparent account of the study being reported; that no important aspects of the study have been omitted; and that any discrepancies from the study as originally planned (and, if relevant, registered) have been explained.

Data sharing: Anonymised data are available on request.

This is an Open Access article distributed in accordance with the Creative Commons Attribution Non Commercial (CC BY-NC 4.0) license, which permits others to distribute, remix, adapt, build upon this work non-commercially, and license their derivative works on different terms, provided the original work is properly cited and the use is noncommercial. See: http://creativecommons.org/licenses/by-nc/4.0/.
1 Goldenberg NA, Spyropoulos AC, Halperin JL, et al, Antithrombotic Trials Leadership and Steering Group. Improving academic leadership and oversight in large industry-sponsored clinical trials: the ARO-CRO model. Blood 2011;117:2089-92 doi:10.1182/blood-2010-09-308858

2 Fox L, Toms C, Kernaghan S, Snowdon C, Bliss JM. Conducting noncommercial international clinical trials: the ICR-CTSU experience. Trials 2017;18:440. doi:10.1186/s13063-017-2176-0

3 Fong EA, Wilhite AW. Authorship and citation manipulation in academic research. PLoS One 2017;12:e0187394. doi:10.1371/journal.pone.0187394

4 Wislar JS, Flanagin A, Fontanarosa PB, Deangelis CD. Honorary and ghost authorship in high impact biomedical journals: a cross sectional survey. BMJ 2011;343:d6128. doi:10.1136/bmj.d6128 5 Kneller R, Mongeon M, Cope J, Garner C, Ternouth P. Industryuniversity collaborations in Canada, Japan, the UK and USA--with emphasis on publication freedom and managing the intellectual property lock-up problem. PLoS One 2014;9:e90302. doi:10.1371/journal pone.0090302

6 Mello MM, Clarridge BR, Studdert DM. Academic medical centers' standards for clinical-trial agreements with industry. N Engl J Med 2005;352:2202-10. doi:10.1056/NEIMsa044115

7 Kasenda B, von Elm E, You JJ, et al. Agreements between Industry and Academia on Publication Rights: A Retrospective Study of Protocols and Publications of Randomized Clinical Trials. PLoS Med 2016;13:e1002046. doi:10.1371/journal. pmed.1002046

8 Schulman KA, Seils DM, Timbie JW, et al. A national survey of provisions in clinical-trial agreements between medical schools and industry sponsors. N Engl J Med 2002;347:1335-41. doi:10.1056/NEJMsa020349

9 Bodenheimer T. Uneasy alliance--clinical investigators and the pharmaceutical industry. N Engl J Med 2000;342:1539-44. doi:10.1056/NEJM200005183422024

10 Rochon PA, Sekeres M, Hoey J, et al. Investigator experiences with financial conflicts of interest in clinical trials. Trials 2011;12:9. doi:10.1186/1745-6215-12-9

11 Moses H3rd, Matheson DH, Cairns-Smith S, George BP, Palisch C, Dorsey ER. The anatomy of medical research: US and international comparisons. JAMA 2015;313:174-89. doi:10.1001/jama.2014.15939

12 Lundh A, Krogsbøll LT, Gøtzsche PC. Sponsors' participation in conduct and reporting of industry trials: a descriptive study. Trials 2012;13:146. doi:10.1186/1745-6215-13-146

13 Clarivate Analytics. 2015 Journal Citation Reports $₫$ Science Edition. 2017. http://jcr.incites.thomsonreuters.com/JCRJournalHomeAction. action.

14 van Lent M, Overbeke J, Out HJ. Recommendations for a uniform assessment of publication bias related to funding source. BMC Med Res Methodol 2013;13:120. doi:10.1186/1471-2288-13-120

15 International Committee of Medical Journal Editors. Recommendations for the conduct, reporting, editing, and publication of scholarly work in medical journals. 2017 http://www.icmje.org/icmje-recommendations.pdf.

16 Del Greco L, Walop W. Questionnaire development: 1. Formulation. CMAl 1987;136:583-5.

17 Edwards PJ, Roberts I, Clarke MJ, et al. Methods to increase response to postal and electronic questionnaires. Cochrane Database Syst Rev 2009;3:MR000008.

18 Lundh A, Krogsbøll LT, Gøtzsche PC. Access to data in industry sponsored trials. Lancet 2011;378:1995-6. doi:10.1016/S0140-6736(11)61871-0

19 Gøtzsche PC, Hróbjartsson A, Johansen HK, Haahr MT Altman DG, Chan AW. Constraints on publication rights in industry-initiated clinical trials. JAMA 2006;295:1645-6. doi:10.1001/jama.295.14.1645

20 Gøtzsche PC, Hróbjartsson A, Johansen HK, Haahr MT, Altman DG, Chan AW. Ghost authorship in industry-initiated randomised trials. PLoS Med 2007;4:e19. doi:10.1371/journal.pmed.0040019

21 Ross JS, Hill KP, Egilman DS, Krumholz HM. Guest authorship and ghostwriting in publications related to rofecoxib: a case study of industry documents from rofecoxib litigation. JAMA 2008;299: 1800-12. doi:10.1001/jama.299.15.1800

22 Forsyth SR, Odierna DH, Krauth D, Bero LA. Conflicts of interest and critiques of the use of systematic reviews in policymaking: an analysis of opinion articles. Syst Rev 2014;3:122. doi:10.1186/2046-4053-3-122

23 Cook D, Kaminski K. Competing interest disclosures compared with industry payments reporting among highly cited authors in clinical medicine. International Congress on Peer Review and Scientific Publication 2017, 12 September 2017. American Medical Association, 2017.

24 Rasmussen K, Schroll J, Gøtzsche PC, Lundh A. Under-reporting of conflicts of interest among trialists: a cross-sectional study. J $R$ Soc Med 2015:108:101-7. doi:10.1177/0141076814557878 
25 Glaser BE, Bero LA. Attitudes of academic and clinical researchers toward financial ties in research: a systematic review. Sci Eng Ethics 2005;11:553-73. doi:10.1007/s11948-005-0026-z

26 Smyth RM, Kirkham JJ, Jacoby A, Altman DG, Gamble C, Williamson PR. Frequency and reasons for outcome reporting bias in clinical trials: interviews with trialists. BM/ 2011:342:c7153. doi:10.1136/bmj.c7153

27 Cunningham CT, Quan H, Hemmelgarn B, et al. Exploring physician specialist response rates to web-based surveys. BMC Med Res Methodol 2015;15:32. doi:10.1186/s12874-015-0016-z

28 Schroll JB, Bero L, Gøtzsche PC. Searching for unpublished data for Cochrane reviews: cross sectional study. BMJ 2013;346:f2231. doi:10.1136/bmj.f2231
29 Schulz KF, Altman DG, Moher D, CONSORT Group. CONSORT 2010 statement: updated guidelines for reporting parallel group randomised trials. BMJ 2010;340:c332. doi:10.1136/bmj.c332

30 Lugtenberg M, Burgers JS, Westert GP. Effects of evidence-based clinical practice guidelines on quality of care: a systematic review. Qual Saf Health Care 2009;18:385-92. doi:10.1136/qshc.2008.028043

31 Cook DJ, Guyatt GH, Laupacis A, Sackett DL. Rules of evidence and clinical recommendations on the use of antithrombotic agents. Chest 1992;102(Suppl):305S-11S.

\section{Appendix 1-7}

